\title{
VERIFICATION AND VALIDATION STUDY FOR A HYDROFOIL MOUNTED ON A PLATE
}

\author{
Costel Ungureanu \\ ${ }^{1}$ University "Dunarea de Jos" of Galati, \\ Faculty of Naval Architecture, Galati, Domneasca \\ Street, No. 47, 800008, Romania, \\ E-mail:costel.ungureanu@ugal.ro \\ ${ }^{2}$ Bureau Veritas Romania \\ 165 Splaiul Unirii Street, TN Offices, 3rd Building, \\ B Wing, 5th Floor, 3rd District, 030133, \\ Bucharest, Romania, \\ E-mail:costel.ungureanu@bureauveritas.com
}

\author{
Costel Iulian Mocanu \\ University "Dunarea de Jos" of Galati, \\ Faculty of Naval Architecture, Galati, Domneasca \\ Street, No. 47, 800008, Romania, \\ E-mail:costel.mocanu@ugal.ro
}

\begin{abstract}
The junction flow between an hydrofoil and a plate is manifested by the generation of vortex structures as a result of the interaction between the boundary layer on the plate and the boundary layer on the profile. Two benchmark tests have been identified in the literature: one for the flat plate, and the other for the NACA 0012 profile. The Verification and Validation study for both hydrofoil and flat plate was performed by testing all turbulence model implemented in Ansys Fluent and results are compared with the experimental ones.
\end{abstract}

Keywords: verification and validation, error, uncertainty, flat plate, drag coefficient

\section{INTRODUCTION}

The significant development of numerical methods for solving engineering problems has inevitably led to the need to assess the credibility of the results. This process is known in the literature as Verification and Validation. According to Roache (1997), verification is a purely mathematical process that proves those equations are solved correctly and quantifies numerical errors, while validation is a physics-based scientificengineering process that proves that correct equations are solved and estimates errors due to modeling.

The first public statement on assessing the accuracy of numerical solutions belongs to the editorial policy of the ASME Journal of Fluids Engineering (Roache et al., 1986), and revised by Freitas (1993). Similar re- quirements have been adopted by the AIAA Journal (AIAA, 1994), ASME Journal of Heat Transfer (Editorial Committee, 1993) and International Journal for Numerical Methods in Fluids (Gresho and Taylor, 1994). All editorial policies had in common two basic requirements for numerical studies to be published. Each numerical problem had to be set out clearly and completely so that any interested reader could reproduce the studies, and the numerical results together with the calculation program were accompanied by estimates of the accuracy of the results. All studies had to specify iterative and spatial errors as well as those due to temporal discretization, but without evaluating discretization errors. But in 2008 the ASME Journal of Fluid Engineering (Celik et al., 2008) was the first journal to publish in its 
editorial policy a five-step procedure for determining numerical uncertainty.

Although the literature is extensive on this topic, Oberkampf (2010), Roache (1997, 1998), Stern and Coleman (1999, 2008), Eça and Hoekstra (2012), there is no universal procedure for complex turbulent applications in fluid dynamics. As proof are the workshops organized by Luis Eça and Martin Hoekstra in Lisbon, in 2004, 2006 and 2008, during which uncertainties were discussed and analyzed on three levels: verification of calculation programs, verification of the solution, and validation of the solution based on cases of two-dimensional, turbulent, stationary flow of incompressible fluids existing in the ERCOFTAC database. The series of workshops continues in 2010, during the conference organized by ECCOMAS (European Community of Computational Methods in Applied Sciences), in a section dedicated entirely to verification and validation of numerical results, culminating in 2012 with the symposium dedicated to verification and validation by the American Society of ASME-American Society of Mechanical Engineers. Stern et al. (1999) are the first to propose a pragmatic approach for determining the errors and uncertainties associated with numerical simulations in naval hydrodynamics. The first guide with recommendations for verification and validation in CFDs appeared in 1998 at the American Institute of Aeronautics and Astronautics (AIAA). In 2000, the best practice guide in CFD issued by ERCOFTAC appears, which deals with the problem of evaluating the errors of numerical simulations. However, discussions and methodologies for estimating calculation errors and their associated uncertainties in the CFD have reached a certain level of maturity with the publication in 2009 by ASME of the complete guide for validation and verification in solving problems in fluid mechanics and thermal transfer.

The flow around the junctions between a profile and a plate is manifested by the generation of vortex structures as a result of the interaction between the boundary layer on the plate and the boundary layer on the profile. Two benchmark tests have been identified in the literature: one for the flat plate, and the other for the NACA 0012 profile. As a result, the study for the validation of the immersion junction calculation methodology will have two components: on the one hand, the flow on the plate will be studied, flat in relation to the results obtained by Yang and Voke (1993), and centralized in case 73 of the ERCOFTAC database, and on the other hand will study the flow around the aerodynamic profile NACA0012 in relation to the results obtained by NASA Langley Research Center- Turbulence Modeling Resource.

\section{FLOW AROUND NACA 0012 HYDROFOIL}

\subsection{Benchmark}

For the validation of the viscous flow around the aero-hydrodynamic profile NACA 0012 was chosen the study presented by NASA Langley Research Center and presented on Turbulence Modeling resources, in case of flow without angle of attack at Reynolds number $3 \times 10^{6}$.

\subsection{Verification and Validation}

Three O-H grids, with grid coarsening ratios, $r_{i j}=\sqrt{2}$, were generated, according to ASME V\&V 20 (2009), on a circular calculation domain with a minimum radius of three chord lengths. The generation of the fine grid is performed by hyperbolic extrusion, starting from the profile, discretized with 150 nodes, with the cell growth ratio in the boundary layer, $r=1.1$, obtaining the two-dimensional O type having $301 \times 94$ cells. On the vertical direction of the computational domain, or on the width of the profile, the basic grid is extruded on a unit length with 80 equidistant nodes, finally obtaining the grid with $301 \times 94 \times 80$ knots. 


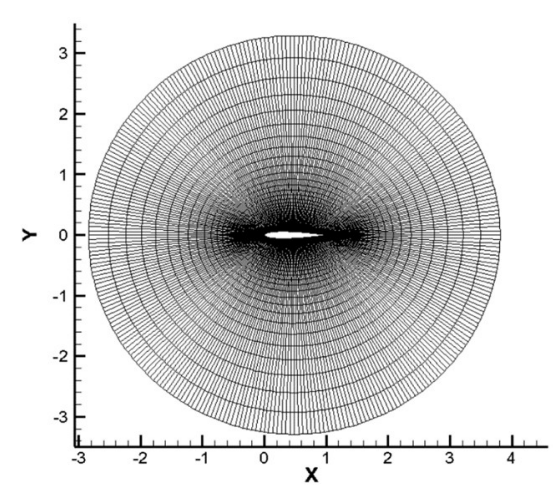

Fig.1. O-H grid around NACA 0012, top view

To keep the same solving conditions in the boundary layer, the grids were generated starting from the same distance to the dimensionless wall, $\mathrm{y}+=1$.

Table 1 presents the grids generated in the study, fine, medium and coarse.

\begin{tabular}{cccc}
\multicolumn{4}{c}{ Table 1. Generated grids } \\
\hline Grid & Fine (1) & Medium (2) & Coarse (3) \\
\hline $\mathbf{N}_{\mathbf{i}}$ & 2204100 & 771680 & 269100 \\
$\sum \mathbf{V}_{\mathbf{i}}$ & 34.3 & 34.3 & 34.3 \\
{$\left[\mathbf{m}^{3}\right]$} & & $4.441 \mathrm{E}-05$ & $1.274 \mathrm{E}-04$ \\
$\mathbf{h}_{\mathbf{i}}\left[\mathbf{m}^{3}\right]$ & $1.555 \mathrm{E}-05$ & 1,414 & \\
$\mathbf{r 2 1}$ & & & 1,414 \\
$\mathbf{r 3 2}$ & & & \\
\hline
\end{tabular}

The numerical simulations were performed with Ansys Fluent v12, with the pressure-based solver, related to the incompressible flow. The inlet boundary was defined as "velocity inlet", the outlet boundary was defined as presure outlet and the no slip condition on hydrofoil. The top and bottom boundaries were declared as "symmetry" type borders. All calculations were performed with the double-precision solver, and for convergence the value $10^{-6}$ was imposed for residues. As a result, rounding and iterative errors were neglected, being considered only discretization errors in determining numerical errors. The pressure and velocity equations were coupled with the SIMPLEC scheme, the pressure was calculated with the
Standard scheme. For the stability of the solver, convergence was first obtained using first-order upwind schemes. (FOU) for impulse and turbulent quantities and then to achieve convergence again using secondorder upwind schemes (SOU).

All steady-state turbulence models available in Ansys v12 were tested, and the results of the drag coefficient together with the discretization error and uncertainty are presented in Table 2. The lowest values of the discretization uncertainty are obtained with the $k-\varepsilon$ Standard and Reynolds stress modelLinear pressure strain models, around 3\%, and the highest values are obtained with the Reynolds stress model with the Low Reynolds option.

Table 2. Drag coefficient grid errors and uncertainties

\begin{tabular}{|c|c|c|c|c|c|}
\hline $\begin{array}{l}\text { Turb. } \\
\text { mod. }\end{array}$ & (1) & (2) & (3) & $\begin{array}{c}\boldsymbol{\delta}_{\mathbf{G}} \\
{[\%]}\end{array}$ & $\begin{array}{c}\mathbf{U}_{\mathbf{G}} \\
{[\%]}\end{array}$ \\
\hline SA1 & 7.426 & 8.117 & 9.026 & 29.40 & 36.75 \\
\hline SA2 & 7.363 & 8.055 & 8.964 & 29.78 & 37.22 \\
\hline $\mathrm{k} \varepsilon \mathrm{S}$ & 1.086 & 1.998 & 1.658 & 49.94 & 62.42 \\
\hline $\begin{array}{l}\mathrm{k} \varepsilon \\
\mathrm{RNG}\end{array}$ & 6.497 & 9.475 & 1.466 & 61.87 & 77.33 \\
\hline $\mathrm{K} \varepsilon \mathrm{R}$ & 4.986 & 5.823 & 6.948 & 48.89 & 61.11 \\
\hline $\mathrm{k} \omega \mathrm{S}$ & 1.724 & 2.061 & 4.558 & 3.06 & 3.82 \\
\hline $\begin{array}{l}\text { K } \omega \\
\text { SST }\end{array}$ & 7.102 & 6.121 & 7.423 & 42.30 & 52.87 \\
\hline $\begin{array}{l}\mathrm{k} \omega \\
\text { SST- } \\
\text { TF }\end{array}$ & 6.263 & 4.372 & 5.365 & 33.40 & 41.75 \\
\hline $\begin{array}{l}\text { RSM- } \\
\text { LPS- } \\
\text { EWT }\end{array}$ & 4.886 & 5.738 & 1.254 & 2.50 & 3.12 \\
\hline $\begin{array}{l}\text { RSM } \\
\text {-LR }\end{array}$ & 4.520 & 5.315 & 6.302 & 72.65 & 90.82 \\
\hline
\end{tabular}

Where

Drag coefficient values presented are multiplied with $10^{3}$. The first column contains the turbulence models taken into account.

The results obtained with the fine grid are compared with those obtained by Ladson, 1988, Gregory and O'Reilly, 1970, Abbott and von Doenhoff, 1959, and Jespersen et al. 2016, and together with the validation error and the validation uncertainty, are presented in table 3 . 
Table 3. Comparison error uncertainty

\begin{tabular}{|c|c|c|c|c|c|}
\hline Turb & Fine & Exp. & $\begin{array}{c}\mathrm{E} \\
{[\%]}\end{array}$ & $\begin{array}{l}\text { Usn } \\
{[\%]}\end{array}$ & $\begin{array}{c}\mathrm{Ue} \\
{[\%]}\end{array}$ \\
\hline SA1 & 7.426 & 8.255 & -10.04 & 36.75 & 38.09 \\
\hline $\mathrm{SA} 2$ & 7.363 & 8.255 & -10.80 & 37.21 & 38.75 \\
\hline $\mathrm{k} \varepsilon \mathrm{S}$ & 1.086 & 8.255 & 31.53 & 62.42 & 69.93 \\
\hline $\begin{array}{l}\mathrm{k} \varepsilon \\
\mathrm{RNG}\end{array}$ & 6.497 & 8.255 & -21.29 & 77.33 & 80.21 \\
\hline $\mathrm{K} \varepsilon \mathrm{R}$ & 4.986 & 8.255 & -39.60 & 61.10 & 72.81 \\
\hline $\mathrm{k} \omega \mathrm{S}$ & 1.724 & 8.255 & 108.8 & 3.82 & 108.87 \\
\hline $\begin{array}{l}\mathrm{K} \omega \\
\mathrm{SST} \\
\mathrm{k} \omega\end{array}$ & 7.102 & 8.255 & -13.96 & 52.86 & 54.68 \\
\hline $\begin{array}{l}\text { SST- } \\
\text { TF }\end{array}$ & 6.263 & 8.255 & -24.12 & 41.75 & 48.22 \\
\hline $\begin{array}{l}\text { RSM- } \\
\text { LPS- } \\
\text { EWT }\end{array}$ & 4.886 & 55 & 80 & .12 & 40.92 \\
\hline $\begin{array}{l}\text { RSM } \\
\text {-LR }\end{array}$ & 4.520 & 8.255 & -45.24 & 90.81 & 101.46 \\
\hline
\end{tabular}

All drag coefficients are expressed multiplied by $10^{3}$.

If the $k-\omega$ standard model is excluded from the error analysis, it is observed that the turbulence model with an equation, Spalart Allmaras, with both options, Vorticity based and Strain/Vorticity-based, produces the result closest to the experimental value, within $10 \%$, and the biggest differences are obtained with the Reynolds stress model with the Low Reynolds option. Regarding the validation uncertainty, the models that obtain the values minimum, $38 \%$, and maximum, $101 \%$, are kept.

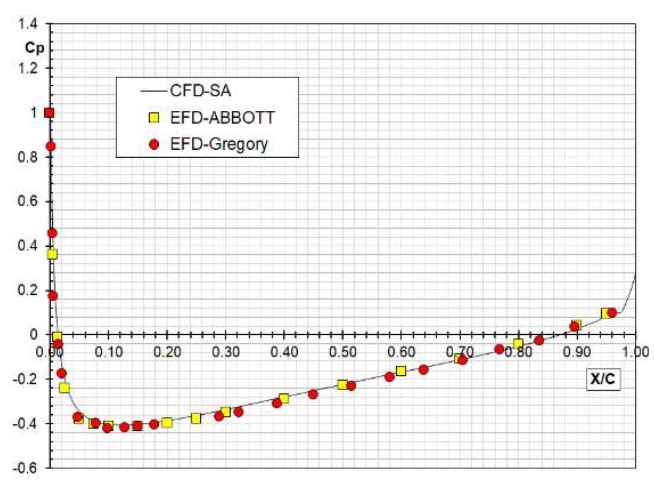

Fig.2. Pressure coefficient distribution on the hydrofoil
The following is a comparison between the numerically calculated pressure coefficient and the experimentally measured one by Abbot (1959) and Gregory (1970) but also a comparison between the friction coefficient calculated with Fluent and the friction coefficient calculated with the CFL3D solver, belonging to NASA.

It can be observed good correlation between the numerical curve with the experimental data of the pressure coefficient.

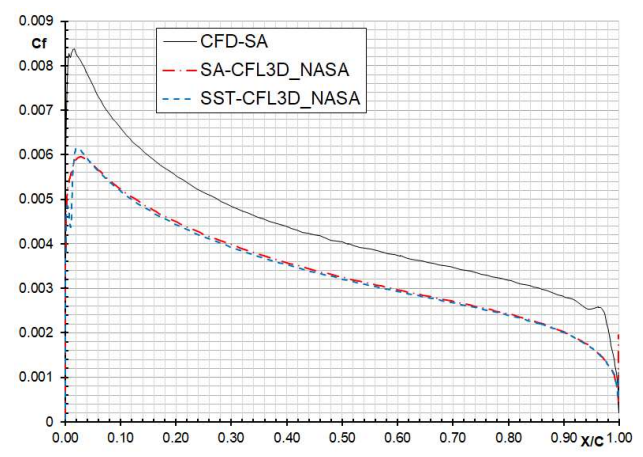

Fig.3. Skin friction coefficient distribution on the hydrofoil

The skin coefficient of friction if qualitatively the distribution is similar to that identified in the literature, quantitatively, the Spalart Allmaras model, calculated with Ansys Fluent overestimated the values of shear stresses by about $25 \%$.

\section{FLOW OVER FLAT PLATE}

\subsection{Benchmark}

The study considered for the validation of the viscous flow on the flat plate is the numerical experiment performed by Yang and Voke in 1993 and made public with the number C.73 from the ERCOFTAC database, Classic collection. The numerical study consists in the calculation using LES (Large Eddies Simulation) at parallel flow with a flat plate measuring $300 \mathrm{~mm} \times 20 \mathrm{~mm}$, without pressure gradient, with 5\% turbulence imposed and a speed of $9.6 \mathrm{~m} / \mathrm{s}$. 


\subsection{Verification and Validation}

Taking into account the peculiarity of the flow, three two-dimensional grids, $300 \mathrm{~mm} \times 30 \mathrm{~mm}$, with grid coarsening ratios, $r_{i j}=\sqrt{2}$, were generated, according to ASME V\&V 20 (2009). To keep the same solving conditions in the boundary layer, the grids were generated starting from the same the dimensionless wall distance, $\mathrm{y}^{+}=1$.

Table 4. Generated grids

\begin{tabular}{cccc}
\hline Grid & Fine (1) & Medium (2) & Coarse (3) \\
\hline $\mathbf{N}_{\mathbf{i}}$ & 8000 & 2000 & 500 \\
$\sum \mathbf{A}_{\mathbf{i}}\left[\mathbf{m}^{2}\right]$ & 0,009 & 0,009 & 0,009 \\
$\mathbf{h}_{\mathbf{i}}\left[\mathbf{m}^{2}\right]$ & $1,125 \mathrm{E}-06$ & $4,500 \mathrm{E}-06$ & $1,800 \mathrm{E}-05$ \\
$\mathbf{r 2 1}$ & & 1.414 & \\
$\mathbf{r 3 2}$ & & & 1.414 \\
\hline
\end{tabular}

The numerical simulations were performed with Ansys Fluent v12, with the pressure-based solver, related to the incompressible flow. The inlet boundary was defined as "velocity inlet, the outlet boundary was defined as presure outlet. The wall condition was applied to the plate, and the symmetry condition was applied to the upper border. All calculations were performed with the double precision solver, and for convergence the value $10^{-6}$ was imposed for residues. As a result, rounding and iterative errors were neglected, being considered only discretization errors in determining numerical errors.

All steady-state turbulence models available in Ansys v12 were tested, and the values of the drag coefficient together with the discretization error and uncertainty are presented in Table 3.10. Although it is not a model of turbulence, the case of laminar flow was also considered, as the transition from laminar flow to turbulent flow is also studied. The models of turbulence in the family are better suited to grids with $y+>30$. As a result, in order to be able to be used on the study grid, at $\mathrm{y}+=1$, the wall functions were activated through the "Enhanced wall treatment" option.
Table 5. Discretization error and uncertainty for the drag coefficient

\begin{tabular}{cccccc}
\hline Turb & $\mathrm{G} 1$ & $\mathrm{G} 2$ & $\mathrm{G} 3$ & $\begin{array}{c}\delta_{\mathrm{G}} \\
{[\%]}\end{array}$ & $\begin{array}{c}\mathrm{U}_{\mathrm{G}} \\
{[\%]}\end{array}$ \\
\hline Lam & 3.224 & 3.206 & 3.118 & 0.1370 & 0.1713 \\
\hline $\mathrm{SA}$ & 6.905 & 6.964 & 6.970 & 0.0902 & 0.1127 \\
\hline $\mathrm{k} \varepsilon \mathrm{S}$ & 7.016 & 7.036 & 7.074 & 0.2931 & 0.3663 \\
$\mathrm{k} \varepsilon$ & 7.007 & 7.025 & 7.055 & 0.3517 & 0.4397 \\
$\mathrm{RNG}$ & & & & \\
$\mathrm{K} \varepsilon \mathrm{R}$ & 7.021 & 7.043 & 7.088 & 0.2932 & 0.3665 \\
\hline $\begin{array}{c}\mathrm{k} \omega \mathrm{S} \\
\mathrm{K} \omega\end{array}$ & 6.614 & 6.548 & 6.004 & 0.1388 & 0.1735 \\
$\begin{array}{c}\mathrm{SST} \\
\mathrm{k} \omega\end{array}$ & 6.348 & 6.594 & 6.124 & 4.2711 & 5.3389 \\
$\begin{array}{c}\mathrm{SST}- \\
\mathrm{TF}\end{array}$ & 6.713 & 6.594 & 6.124 & 0.5959 & 0.7448 \\
\hline $\begin{array}{c}\text { RSM- } \\
\text { LPS- } \\
\text { EWT }\end{array}$ & 6.630 & 6.716 & 6.990 & 0.6042 & 0.7553 \\
$\begin{array}{c}\text { RSM } \\
\text {-LR }\end{array}$ & 6.180 & 5.805 & 5.390 & 6.0764 & 7.5955 \\
$\begin{array}{c}\text { RSM- } \\
\text { LR- } \\
\text { TF }\end{array}$ & 5.243 & 4.874 & 4.435 & 7.0378 & 8.7972 \\
\hline
\end{tabular}

where G1, G2 and G3 are the fine, medium and coarse grid. Drag coefficient values presented are multiplied with $10^{3}$. The first column contains the turbulence model taken into account.

\begin{tabular}{|c|c|c|c|c|c|}
\hline Turb & Fine & Exp. & $\begin{array}{c}\mathrm{E} \\
{[\%]}\end{array}$ & $\begin{array}{l}\text { Usn } \\
{[\%]}\end{array}$ & $\begin{array}{c}\mathrm{Ue} \\
{[\%]}\end{array}$ \\
\hline Lam & 3.224 & 2.970 & -8.570 & 0.171 & 8.569 \\
\hline SA & 6.905 & 6.883 & -0.318 & 0.113 & 0.297 \\
\hline $\mathrm{k} \varepsilon \mathrm{S}$ & 7.016 & 6.883 & -1.940 & 0.366 & 1.905 \\
\hline $\begin{array}{c}\mathrm{k} \varepsilon \\
\mathrm{RNG}\end{array}$ & 7.007 & 6.883 & -1.804 & 0.440 & 1.750 \\
\hline $\mathrm{K} \varepsilon \mathrm{R}$ & 7.021 & 6.883 & -2.001 & 0.366 & 1.967 \\
\hline $\mathrm{k} \omega \mathrm{S}$ & 6.614 & 6.883 & 3.908 & 0.174 & 3.904 \\
\hline $\begin{array}{c}\mathrm{K} \omega \\
\mathrm{SST} \\
\mathrm{k} \omega\end{array}$ & 6.348 & 6.883 & 7.767 & 5.339 & 5.642 \\
\hline $\begin{array}{c}\text { SST- } \\
\text { TF } \\
\end{array}$ & 6.713 & 6.883 & 2.467 & 0.745 & 2.351 \\
\hline $\begin{array}{l}\text { RSM- } \\
\text { LPS- } \\
\text { EWT }\end{array}$ & 6.630 & 6.883 & 3.680 & 0.755 & 3.601 \\
\hline $\begin{array}{l}\text { RSM } \\
\text {-LR }\end{array}$ & 6.180 & 6.883 & 10.206 & 7.596 & 6.816 \\
\hline $\begin{array}{l}\text { RSM- } \\
\text { LR- } \\
\text { TF }\end{array}$ & 5.243 & 6.883 & 23.818 & 8.797 & 22.134 \\
\hline
\end{tabular}


All drag coefficients are expressed multiplied by $10^{3}$.

It is observed that the turbulence model with an equation, Spalart Allmaras produces the result closest to the experimental value, and the highest values of the validation error are obtained with the Reynolds Stress Model with the option Low Reynolds transitional flow.

Figure 4 compares the experimental mean velocity profile with numerical values calculated with Spalart-Allmaras, $k-\varepsilon$ Realizable and $k-\omega$ SST-TF models, at various sections in the calculation field. Differences are observed between the numerical and experimental values due to the intersection of the wall boundary with the inlet boundary and the transition zone of the laminar to turbulent flow. When the flow becomes turbulent, the numerical speed profile overlaps with the experimental one, within the limit of $12 \%$.

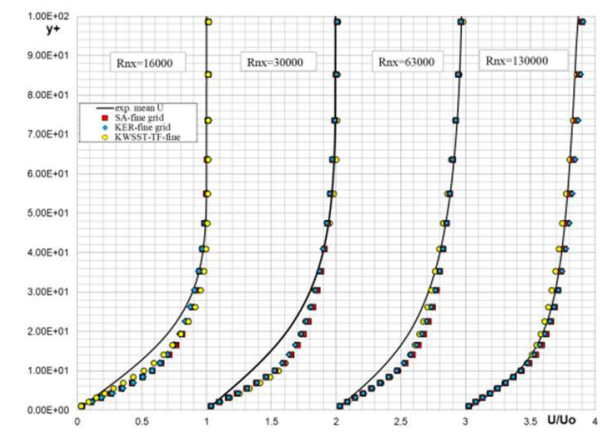

Fig.4. Mean velocity inside boundary layer

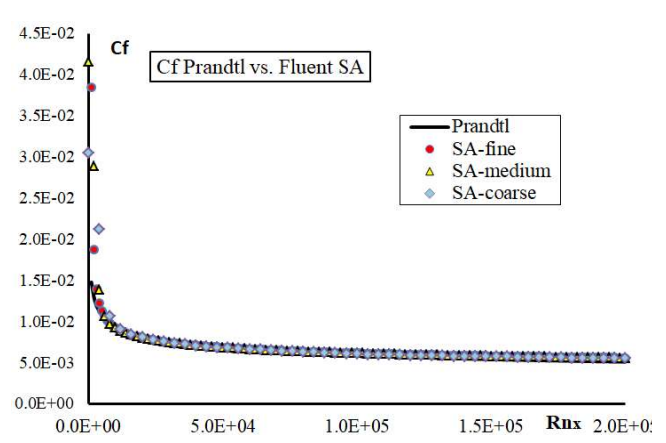

Fig.5. Skin friction coefficient, comparison SA (Spalart-Allmaras) vs Prandtl
Next, the comparison between the computed skin friction coefficient and the theoretical one, Prandtl for turbulent flow is presented. It can be qualitatively concluded that the curves are in good agreement.

\section{CONCLUDING REMARKS}

Following studies of viscous flow on the flat plate and around the aero-hydrodynamic profile NACA0012 and validations with results from the literature it can be concluded that the Spalart Allmaras turbulence model is promising for the study of the flow around junctions formed by an hydrofoil mounted on a plate, where a single equation is a great advantage in terms of hardware resources and computation time.

\section{Acknowledgements}

This research study was performed in the frame of Multidisciplinary Research Platform ReForm Dunarea de Jos University of Galati - The Naval Architecture Research Center.

\section{REFERENCES}

[1]. Roache, P. J, "Quantification of uncertainty in computational fluid dynamics", Annu. Rev. Fluid. Mech., 29:123-60, 1997;

[2]. Roache, P. J., Kirti, N. G., \& White, F. M., "Editorial policy statement on the control of numerical accuracy", Journal of Fluids Engineering, 108, 2, 1986;

[3]. Freitas, C.J., "Engineering editorial policy statement on the control of numerical accuracy". ASME editorial board, 1993;

[4]. AIAA, "Editorial Policy statement on numerical accuracy and experimental uncertainty", American Institute of Aeronatics and Astronautics, 32, 3, 1994;

[5]. ASME, "Statement upon the Control of Numerical Accuracy, Jornal of Fluids Engineering", ASME, 115, 339-340, 1993;

[6]. Gresho, P.M. \& Taylor, C., "Editorial, International Journal of Numerical Methods in Fluids", 19, iii, 1994;

[7]. Celik, I. B., Ghia, U., Roache, P. J., Freitas, C. J., Coleman, H.W., and Raad, P. E., , "Procedure for Estimation and Reporting of Uncertainty due to Discretization 
in CFD Applications", ASME J. Fluids Eng., 130(7), p. 078001, 2008;

[8]. Oberkampf, W.L., Roy, C.J., "Verification and Validation in scientific computing", Cambridge University Press, 2010;

[9]. Oberkampf, W.L., Trucano, T.G., Hirsch, C., "Verification, Validation, and Predictive Capability in Computational Engineering and Physics", SAND REPORT, 2003.

[10]. Oberkampf, W.L., "Verification and Validation in Computational Simulation", Sandia National Laboratories, 2004.

[11]. Oberkampf, W.L., "Perspectives on Verification, Validation, and Uncertainty Quantification", SIAM Conference on Computational Science and Engineering, Miami, Florida, March 2 - 6, 2009.

[12]. Oberkampf, W.L., Roy, C.J., "Verification and Validation in scientific computing", Cambridge University Press, 2010.

[13]. Oberkampf, W.L., "Oberkampf, W.L., "Practical and Technical Challenges in Verification and Validation", Institute for Computing in Science, Verification, Validation and Uncertainty Quantification, August 613, 2011.

[14]. Oberkampf, W.L., "Practical and Technical Challenges in Verification and Validation", American Society of Mechanical Engineers, Verification and Validation Symposium, Las Vegas, Nevada, May 2 - 4, 2012.

[15]. Roache, P. J., "Verification and Validation in Computational Science and Engineering", Hermosa Publishers, 1998;

[16]. Roache, P. J., "Procedure for Estimation and Reporting of Uncertainty Due to Discretization in CFD Applications", Journal of Fluid Engineering, ASME, 2008;

[17]. Roache, P. J., "Fundamentals of Verification and Validation", Hermosa Publishers, 2009;

[18]. Stern, F., et.al., "Verification and Validation of CFD simulations", Report no. 407, Iowa Institute of Hydraulic Research, 1999;

[19]. Coleman, H.W., "An Overview of ASME V\&V 20: Standard for Verification and Validation in Computational Fluid Dynamics and Heat Transfer", 3rd Workshop on CFD Uncertainty Analysis, Lisbon, October, 2008;

[20]. Eça, L., Hoekstra M., Roache P.J., "Verification of Calculations: an Overview of the Lisbon Workshop", AIAA Paper 4728,
AIAA Computational Fluid Dynamics Conference, Toronto, June 2005;

[21]. Eça., L., Hoekstra, M., "On the Influence of the Iterative Error in the Numerical Uncertainty of Ship Viscous Flow Calculations", 26th Symposium on Naval Hydrodynamics, Rome, Italy, 17-22 September, 2006;

[22]. Eça., L., Hoekstra, M., "Testing Uncertainty Estimation and Validation Procedures in the Flow Around a Backward Facing Step", 3rd Workshop on CFD Uncertainty Analysis, Lisbon, October, 2008;

[23]. Eça., L., Vaz, G., Hoekstra, M., "A Verification and Validation exercise for the flow over a back ward facing step", V European Conference on Computational Fluid Dynamics, ECCOMAS CFD, Lisbon, Portugal,1417 June, 2010;

[24]. Eça., L., Hoekstra, M., Vaz, G., "Code Verification, Solution Verification and Validation in RANS Solvers", OMAE Shanghai, China, June 6-11, 2010.

[25]. Eça., L. and Vaz, G., "Workshop on Verification and Validation of CFD for Offshore Flows", OMAE Rio de Janeiro, Brasil, July $1-6,2012$

[26]. ERCOFTAC, "Best Practice Guidelines for Industrial CFD”, 2000;

[27]. ASME V\&V 20, "Standard for Verification and Validation in Computational Fluid Dynamics and Heat Transfer", 2009;

[28]. Yang, Z., and Voke, P., "Large Eddy Simulation of Transition under Turbulence" in Engineering Turbulence Modelling and Experiments", 2ed, W Rodi and F Martelli, Elsevier Science Amsterdam, 1993.

[29]. Ladson, C. L., Hill, A. S., and Johnson, Jr., W. G., "Pressure Distributions from High Reynolds Number Transonic Tests of an NACA 0012 Airfoil in the Langley 0.3Meter Transonic Cryogenic Tunne"l, NASA TM 100526, 1988;

[30]. Gregory, N. and O'Reilly, C. L., "LowSpeed Aerodynamic Characteristics of NACA 0012 Aerofoil Sections, including the Effects of Upper-Surface Roughness Simulation Hoar Frost", NASA R\&M 3726, 1970;

[31]. Abbot,I.H. \& von Doenhoff, A.E., "Theory of wing sections", Dover Publications, New York, 1959;

[32]. Dennis C. Jespersen, Thomas H. Pulliam, Marissa L. Childs, "OVERFLOW /Turbulence Modeling Resource/Validation 
Results", NASA ;Ames Research Center, 2016.

[33]. Stern, F., et.al., "Verification and Validation of CFD simulations", Report no. 407, Iowa Institute of Hydraulic Research, 1999.

[34]. Stern, F., et.al., "Quantitative V\&V of CFD Simulations and Certification of CFD Codes With Examples", ICHMT International Symposium on Advances in Computational Heat Transfer, Norway, April 19-24, 2004.

[35]. Roy, C.J., "Review of code and solution verification procedures for computational simulation", Journal of Computational Physics, 205, 131-156, 2005.

[36]. Roy, C.J., and Oberkampf, W.L., " $A$ Complete Framework for Verification, Validation, and Uncertainty Quantification in Scientific Computing”, AIAA, 124, 2010.

[37]. Zou, L, Larsson, L., Orych, M., "Verification and validation of CFD predictions for a manoeuvring tanker", $9^{\text {th }}$ International Conference on Hydrodynamics, October, 11-15, 2010.

Paper received on 15 of August, 2020 